\section{Synthetic influenza seeds}

\section{By Amy Donner, Senior Editor}

An international team led by Novartis AG and the J. Craig Venter Institute has improved the speed and accuracy of seed influenza virus production for large-scale vaccine manufacture. ${ }^{1}$ Adoption of the platform could shave weeks off the time needed to generate vaccine in response to a pandemic.

Influenza vaccines typically are developed seasonally from seed viruses representing the year's prevalent or virulent strains. The stocks of seed viruses are produced at centralized facilities working in concert with the World Health Organization and are distributed for vaccine manufacturing via a complex process involving multiple institutions and shipping steps.

The slow distribution of seed virus contributes to the overall inefficient production of influenza vaccine in response to pandemics.

The six-month response time to the $2009 \mathrm{H} 1 \mathrm{~N} 1$ influenza virus-spanning the whole cycle from recognition of the pandemic to vaccine availability-is the best on record, but the swine flu vaccine was still only available to the public after the pandemic had actually peaked.

To accelerate seed virus production, a team led by Philip Dormitzer, Rino Rappuoli and J. Craig Venter took on two major technical barriers to the production of synthetic seed virus: the speed and accuracy of influenza genome synthesis.

Dormitzer is head of virology and research in the U.S. at Novartis' Vaccines and Diagnostics unit. Rappuoli is global head of vaccine research at Novartis Vaccines and Diagnostics, and Venter is founder, chairman and CEO of the J. Craig Venter Institute (JCVI) and founder of Synthetic Genomics Inc.

The collaborators started by adapting protocols developed by JCVI for synthesizing the mouse mitochondrial genome ${ }^{2}$ to the synthesis of genes encoding influenza antigens.

The synthetic process involved production of overlapping and complementary oligonucleotides representing the complete coding sequences for the $1.7 \mathrm{~kb}$ influenza A virus hemagglutinin (HA) and influenza $B$ virus $\mathrm{HA}$ or the $1.5 \mathrm{~kb}$ neuraminidase (NA), which are viral surface proteins that serve as the basis of most approved flu vaccines. Assembly of the segments did not require cloning or sequencing steps.

Initial attempts to assemble viral gene segments based on the mitochondrial protocol yielded only about $3 \%$ of product with the right sequence. Thus, the team worked to improve the assembly protocol by extending the overlap between complementary oligonucleotides, adding an enzymatic error correction step and increasing the number of oligonucleotides assembled at once.
The changes turned the original stepwise series of subassembly processes into a one-step process that produced gene segments with the correct sequence more than $80 \%$ of the time.

The synthetic genomic segments were combined with elements necessary for expression of HA or NA and introduced into Madin-Darby canine kidney (MDCK) cells, a cell line approved for vaccine manufacture.

The seed virus - a recombinant construct containing the viral gene segments with their expression elements in a viral vector-was recovered from cells with up to 1,000-fold higher efficiency than that for a previously described system ${ }^{3}$ and up to 9.9-fold greater HA yield than that for reference strains.

Improving the yield of HA and efficiency of recovery can also shorten the overall time to vaccine generation. Similar results were obtained with at least six influenza strains.

In a simulated pandemic, the synthetic approach yielded seed virus in four days and four hours from receipt of sequence. In contrast, during the 2009 pandemic it took 36 days from identification of the virus to the manufacturers receiving the seed virus, according to a report from the Center for Infectious Disease Research \& Policy (CIDRAP) at the University of Minnesota. ${ }^{4}$

Importantly, when tested in ferrets, the synthetic seed vaccine produced in the simulated pandemic was equivalent to conventional vaccines for antigenicity or induction of an immune response.

Results were published in Science Translational Medicine. The team included researchers from Synthetic Genomics, the Biomedical Advanced Research and Development Authority and the Philipps University of Marburg.

According to Dormitzer, Novartis and the Venter groups are working to automate additional seed-generation steps. Improvements in logistical components of the process-for example, reducing shipping time of samples between collaborating institutionscould further speed up vaccine generation.

\section{One of many steps}

Although the synthetic seed virus production platform provides time savings for one step in vaccine "Rapid synthesis of a seed virus is important, and this is probably how it should be done."

-Steffen Mueller, Codagenix Inc. steps, particularly growing seed virus for large-scale manufacture of vaccine, have a greater impact on the overall response time to a pandemic.

Indeed, the production of sufficient quantities of vaccine from the seed virus generally represents the rate-limiting step, so the rapid method for generating seed virus will still be followed by the slowest, most time-consuming step of the process.

According to Steffen Mueller, president and CSO of Codagenix Inc., "Rapid synthesis of a seed virus is important, and this is probably how it should be done. But with current antigens you need a lot to get an immune response, so you need to grow a lot of virus. Growing massive amounts of virus takes time. This synthetic approach will only shave off two to three weeks of a six-month process." 


\section{ANALYSIS}

One source of inefficiency is reliance upon growing the virus in embryonated chicken eggs with subsequent purification of the vaccine antigen for large-scale vaccine production.

Some companies have received approval for alternative manufacturing platforms that use cell-based systems.

Novartis, which already is implementing MDCK cell lines for influenza vaccine production, is now seeking approval to use the same cells to produce synthetic influenza seed virus. The pharma did not disclose the status of its application.

Weakly immunogenic antigens are another source of inefficiency in the production of vaccine. As a result, large quantities of the antigens are needed for efficacy. According to CIDRAP, most vaccines in clinical trials use the same immunologic approach as current vaccines.

Thus, to dramatically shorten the time it takes to get a vaccine to the public in response to an influenza pandemic, the immunogenicity of antigens needs to be improved.

IP for different portions of the synthetic processes and the cell lines described in the Science Translational Medicine report are owned by Novartis, Synthetic Genomics and MedImmune LLC.

Donner, A. SciBX 6(21); doi:10.1038/scibx.2013.509 published online May 30, 2013

\section{REFERENCES}

1. Dormitzer, P.R. et al. Sci. Transl. Med.; published online May 15, 2013; doi:10.1126/scitranslmed.3006368

Contact: Philip R. Dormitzer, Novartis Vaccines and Diagnostics, Cambridge, Mass. e-mail: philip.dormitzer@novartis.com

2. Gibson, D.G. et al. Nat. Methods 7, 901-903 (2010)

3. Hoffmann, E. et al. Proc. Natl. Acad. Sci. USA 97, 6108-6113 (2000)

4. Osterholm, M.T. et al. The compelling need for game-changing influenza vaccines. (CIDRAP, Oct. 15, 2012)

\section{COMPANIES AND INSTITUTIONS MENTIONED}

Biomedical Advanced Research and Development Authority, Washington, D.C.

Center for Infectious Disease Research \& Policy, Minneapolis, Minn.

Codagenix Inc., Stony Brook, N.Y.

J. Craig Venter Institute, Rockville, Md.

Medlmmune LLC, Gaithersburg, Md.

Novartis AG (NYSE:NVS; SIX:NOVN), Basel, Switzerland

Philipps University of Marburg, Marburg, Germany

Synthetic Genomics Inc., La Jolla, Calif.

University of Minnesota, Minneapolis, Minn.

World Health Organization, Geneva, Switzerland 